\title{
Diskriminierung aus Gründen der sexuellen Ausrichtung: Familienzuschlag, Hinterbliebenenleistung und Beihilfe für die eingetragene Lebenspartnerschaft?
}

\section{Eine Bestandsaufnahme aus europäischer Perspektive' ${ }^{1}$}

\author{
Petra Schott \\ Beamtin in der Europäischen Kommission, Brüssel
}

\section{Die Situation auf europäischer Ebene}

Im Jahr 2000 hat die EU die Richtlinie 2000/78/EG (im Folgenden: die Richtlinie) angenommen, die im Bereich Beschäftigung und Beruf vor Diskriminierungen schützt, die auf dem Alter, einer Behinderung, Religion bzw. dem Glauben oder der sexuellen Ausrichtung beruhen. Die Richtlinie selbst definiert nicht, was unter „sexueller Orientierung“ zu verstehen ist. Sie gilt für alle Personen im öffentlichen und privaten Bereich, einschließlich öffentlicher Stellen, in Bezug auf die Beschäftigungs- und Arbeitsbedingungen, einschließlich der Entlassungsbedingungen und des Arbeitsentgeltes.

Der EuGH hatte sich mehrmals mit Diskriminierungen aus Gründen der sexuellen Ausrichtung auseinanderzusetzen. Neben drei Urteilen ${ }^{2}$ aus früheren Jahren vor der Geltung der Richtlinie hatte erstmals das Urteil Maruko vom 1. April $2008^{3}$ sich mit den Rechten eines eingetragenen Lebenspartners im Versorgungssystem Deutsche Bühnen zu beschäftigen, in dem eine Hinterbliebenenversorgung nur für verheiratete Paare vorgesehen war, nicht jedoch für die registrierte Lebenspartnerschaft. ${ }^{4}$ Das Urteil kam zu dem Ergebnis, dass die Richtlinie einer Regelung entgegensteht, „wonach der überlebende Partner nach Versterben seines Lebenspartners keine Hinterbliebenenversorgung (...) erhält, obwohl die Lebenspartnerschaft nach nationalem Recht Personen gleichen Geschlechts in eine Situation versetzt, die in Bezug auf diese Hinterbliebenenversorgung mit der Situation von Ehegatten vergleichbar ist“. Das Bayerische VG München ist daraufhin mit Urteil vom 30. Oktober 2008 zu dem Ergebnis gekommen, dass Herrn Maruko die Hinterbliebenenrente zustehe. Das Verfahren befindet sich derzeit in der Berufung beim Bayerischen VGH.

Die Umsetzung der Richtlinie 2000/78/EG in Bezug auf die sexuelle Ausrichtung und der Anspruch auf bestimmte Leistungen im öffentlichen Dienst

Die Umsetzung der Richtlinie in Deutschland erfolgte durch das Allgemeine Gleichbehandlungsgesetz (AGG) vom 14. August 2006. Die Ansprüche auf Familienzuschlag, Hinterbliebenenversorgung und Beihilfe der registrierten Lebenspartner(innen) ist sehr uneinheitlich und bedarf deshalb einer genaueren Betrachtung.

\section{Familienzuschlag im Öffentlichen Dienstrecht}

Die Arbeitnehmer(innen) des öffentlichen Rechts erhalten gemäß der Rechtsprechung des $\mathrm{BAG}^{5}$ den Ortszuschlag der
Stufe 2, wenn sie in einer eingetragenen Lebenspartnerschaft leben. Das BAG hat diese Auffassung im Wesentlichen damit begründet, dass der Tarifvertrag dahingehend auszulegen sei.

Uneinheitlich ist hingegen die Situation bei dem Familienzuschlag im Beamtenrecht auf Bundes- und Länderebene. Die Länder Bremen, Berlin, Brandenburg, Hamburg, MecklenburgVorpommern, Rheinland-Pfalz, Saarland und Schleswig-Holstein haben ihre Landesbeamt(inn)en, die in registrierter Partnerschaft leben, mit verheirateten Paaren gesetzlich gleichgestellt, indem sie den Zuschlag gewähren. In den anderen Bundesländern sowie für die Bundesbeamt(inn)en wurde die Gewährung des Zuschlags vom BVerwG und BVerfG bisher abgelehnt. Das BVerwG (Urteil vom 26. Januar 2006) argumentierte, dass eine Gleichstellung zwischen eingetragenen und verheirateten Partner(inne)n im Beamten-Besoldungsrecht vom Gesetzgeber nicht erfolgt sei. Artikel 6 Abs. 1 GG stelle (nur) die Ehe unter einen besonderen Schutz. Die Richtlinie greife nicht ein, denn sie schlösse im Erwägungsgrund 22 an den Familienstand anknüpfende Leistungen von ihrem Geltungsbereich aus. Das BVerfG wies die dagegen eingelegte Verfassungsbeschwerde mit Beschluss vom 20. September 2007 zurück und führte ebenfalls aus, das GG stelle nur die Ehe unter einen besonderen Schutz. Ein weiterer Beschluss des BVerfG vom 6. Mai 2008 kam zu dem Ergebnis, die Versagung des Zuschlags stehe mit der Richtlinie 2000/78/EG in Übereinstimmung, denn der Gesetzgeber habe von einer Gleichstellung der eingetragenen und der verheirateten Paare in diesem Bereich abgesehen.

Das BVerwG hat seine Rechtsprechung zuletzt durch Urteil vom 15. November 2007 mit der Begründung fortgeführt, hinsichtlich der beiden Rechtsinstitute bestehe keine Vergleichbarkeit. Unterschiede bestünden im Familienrecht bei der eigenverantwortlichen Haushaltsführung, dem Gebot der Rücksichtnahme sowie beim Unterhaltsanspruch, im Adoptionsrecht, im Steuerrecht, im Aufenthaltsrecht und im öffentlichen Dienstrecht.

Zusätzlich wird in der Rechtsprechung ${ }^{6}$ damit argumentiert, dass der Familienzuschlag der Förderung heterosexueller Gemeinschaften im Hinblick auf die Fortpflanzung und die Erziehung des eigenen Nachwuchses diene. Zu diesem Anliegen

1 Die Langfassung dieses Aufsatzes kann auf der Internetseite des djb nachgelesen werden.

2 Urteil Grant vom 17.2.1989, C-249/96; Urteil Schweden Rat vom 31.5.2001, C-122/99 und Urteil K.B. vom 7.1.2004, C-117/01.

3 C-267/o6.

4 Aufgrund des Gesetzes über die Eingetragene Lebenspartnerschaft vom 16.2.2001.

5 Z.B. BAG, 29.4.2004, NZA 2005, 57.

6 So OVG Koblenz, 9.3.2009, 2A 11403/o8.OVG. 
könnten homosexuelle Paare nicht in der gleichen Weise beitragen. Auch solle ein gewisser Einkommensausfall wegen der Kindererziehung honoriert werden. Soweit auch in eingetragenen Lebenspartnerschaften Kinder aufgezogen würden, sei der Gesetzgeber nicht gehalten, dies zu fördern.

Würdigung: Soweit von Gerichten ein globaler Vergleich zwischen der Situation in der Ehe und der Situation in einer registrierten Partnerschaft vorgenommen wird, entspricht dies nicht der Rechtsprechung des EuGH, denn in der Maruko-Entscheidung wurde nur auf die Vergleichbarkeit in einem bestimmten Bereich - in dem Fall einzig bei der Hinterbliebenenversorgung - abgestellt und nicht auch auf die Situation zum Beispiel bei Adoptionen oder im Steuerrecht. Die Argumentation, an den Familienstand anknüpfende Leistungen seien vom Geltungsbereich der Richtlinie ausgenommen, ist in dieser Form ebenfalls mit EU-Recht nicht vereinbar. Der EuGH hat im Maruko-Urteil dazu ausgeführt, dass - auch wenn Familienstandsleistungen vom EU-Recht ausgenommen seien -, die Nichtdiskriminierungsvorschriften doch beachtet werden müssten. Soweit es das Argument betrifft, durch den Familienzuschlag würden Fortpflanzung und Erziehung gewürdigt, ist dem zu widersprechen, denn auch kinderlose Paare bekommen den Zuschlag.

\section{Hinterbliebenenleistung}

In $\ 46$ Abs. 4 SGB VI wurde für den Bereich der gesetzlichen Rentenversicherung ein Anspruch für eingetragene Lebenspartner(innen) normiert. Komplizierter ist die Situation im Bereich der betrieblichen Altersversorgung, zu der nach der Rechtsprechung des $\mathrm{EuGH}^{7}$ auch die Beamten- und Arbeitnehmerhinterbliebenenleistungen zählen. Einige Bundesländer haben die Hinterbliebenenleistungen für die Lebenspartnerschaft denen der Ehe angepasst. ${ }^{8}$

Am 14. Februar 2007, also noch vor der EuGH-Entscheidung im Fall Maruko, entschied der $\mathrm{BGH}^{9}$ über die Klage eines registrierten Partners gegen die Versorgungsanstalt des Bundes und der Länder auf Gewährung einer Hinterbliebenenversorgung. Der BGH lehnte die Gewährung der Hinterbliebenenrente $\mathrm{ab}$ und führte aus, dass weder nach der Satzung noch nach dem Grundgesetz oder Europarecht ein Anspruch bestehe.

Am 25. Juli 2007, also ebenfalls noch vor der Maruko-Entscheidung des EuGH, entschied das BVerwG ${ }^{10}$ ablehnend über die Klage eines eingetragenen Partners, dessen verstorbener Partner bei der Bezirksärztekammer Koblenz versichert war. Die Bevorzugung der Ehe sei gerechtfertigt, da diese der Fortpflanzung und Erziehung des Nachwuchses mehr dienen könne als Lebenspartnerschaften. ${ }^{11}$

Gegen das zuerst genannte Urteil des BGH wurde Verfassungsbeschwerde eingelegt, der das BVerfG mit Beschluss vom 7. September $2009^{12}$ unter Rückgriff auf Artikel 3 Abs. 1 GG stattgegeben hat und damit wohl eine Wende in den Urteilen zu diesem Thema eingeleitet haben dürfte. Obwohl die angegriffene Regelung auf Tarifvertrag beruhe, müsse das Gleichbehandlungsgebot gewahrt werden. Für die sexuelle Ausrichtung gelte aufgrund Europarechts (Art. 13 EG, Art. 21 Abs. 1 der Charta der Grundrechte der EU) ein Diskriminierungsverbot. Auch die eingetragene Lebenspartnerschaft sei eine Versorgungsgemeinschaft. Ein Verweis auf den besonderen Schutz der Ehe reiche nicht aus, um unterschiedliche Behandlung zu begründen. Eine Absicht der Tarifpartner, Kinder möglichst mit verheirateten Eltern aufwachsen zu lassen, sei nicht erkennbar. Dem Argument des BGH, der Versorgungsbedarf in Ehen sei höher, weil „typischerweise“ daraus Kinder hervorgingen, wird vom BVerfG entgegengesetzt, dass es nicht in jeder Ehe Kinder gäbe und nicht jede Ehe auf Kinder ausgerichtet sei. Es könne auch nicht mehr davon ausgegangen werden, dass es in Ehen eine Rollenverteilung gäbe, wonach ein Partner deutlich weniger verdiene.

Würdigung: Die Entscheidung des BVerfG ist sehr lesensund begrüßenswert, denn sie räumt mit einigen Argumentationssträngen und einer „Lebenswirklichkeit“ auf, der ein sehr konventionelles Ehemodell zugrunde lag. Die Entscheidung wird zumindest für die Arbeitnehmer(innen) des öffentlichen Dienstes, die in eingetragener Lebenspartnerschaft leben, zu einem Anspruch auf Hinterbliebenenleistung führen. Die Auswirkungen auf das Beamtenrecht, auf den Familienzuschlag und die Beihilfe bleiben abzuwarten. Es scheint jedoch erkennbar, dass die bisherigen Argumente der Gerichte, insbesondere auch die oben dargestellten des BVerwG zum Familienzuschlag, überholt sind.

\section{Beihilfe}

Im Bereich der Beihilfe ist auf Länderebene zum Teil eine Gleichstellung von Ehe und Lebenspartnerschaft vorgenommen worden. ${ }^{13}$ Dabei war Bremen das erste Bundesland, das mit Gesetz vom 23. Oktober $2007^{14}$ diese Erweiterung und Gleichstellung vorgenommen hat.

Soweit diese Gleichstellung nicht erfolgt ist, sind bisher überwiegend versagende Entscheidungen ${ }^{15}$ ergangen, das heißt die Beihilfe wird nicht auf eingetragene Lebenspartner(innen) erstreckt. Mit Beschluss vom 3. Juni $2009^{16}$ hat das BVerwG eine Entscheidung des OVG Koblenz ${ }^{17}$ zu dieser Frage zur Revision angenommen.

\section{Schlussbemerkung}

Abschließend sei festgestellt, dass aufgrund der letzten Entscheidung des BVerfG vom 7. September 2009 zu diesem Themenkreis ein Umschwung in der Rechtsprechung zu erwarten ist, der in die Richtung einer immer weitergehenden Anpassung der Leistungen an die Lebenspartnerschaft und an Ehepaare gehen dürfte.

7 S.z.B. Griesmar, C-366/99.

8 Berlin, Mecklenburg-Vorpommern, Saarland, Brandenburg, Rheinland-Pfalz, Bremen.

9 IV ZR 267/04.

106 C 27/06.

11 Zur Kritik vgl. von Roetteken, Torsten, jurisPr-ArbR 41/2007, Anm. 6.

121 BVR 1164/07.

13 Bremen, Berlin, Brandenburg, Hamburg, Mecklenburg-Vorpommern, Rheinland-Pfalz und Saarland.

$14 \mathrm{GBI}$ Bremen 2007, 480.

15 Z.B. VG Lüneburg vom 14.3.2007, Az. 1 A 230/05 für einen Offizier.

162 B 25/og.

17 Urteil vom 17.12.2008, 10 A 10595/08. 